\title{
TD-LTE - The radio-access solution for IMT-Advanced/TDD
}

\author{
David Astely, Erik Dahlman, Anders Furuskär, Stefan Parkvall
}

\author{
Ericsson \\ 16480 Stockholm, Sweden
}

\begin{abstract}
This paper provides a discussion on the LTE radioaccess technology as defined in 3GPP, with specific focus on its TDD component (TD-LTE). Some specific characteristics of TDD, such as interference between uplink and downlink as well as channel reciprocity, are outlined. A discussion on possible evolution paths for the TD-LTE radio-access technology is also presented. Finally, an overview of the performance and capabilities of TD-LTE, in relation to the requirements on IMTAdvanced, is provided.
\end{abstract}

Keywords:

LTE, LTE-Advanced, TD-LTE, 4G, IMT-Advanced

\section{INTRODUCTION}

Deployment of $4 \mathrm{G}$ mobile-broadband systems based on the LTE radio-access technology [1] is currently ongoing on a broad scale, with the first systems already entering full commercial operation. These systems are based on the first release of LTE, corresponding to 3GPP release 8 . The second LTE release (release 9) was finalized at the end of 2009 and extends the LTE specification with capabilities for e.g. broadcast/multicast services, positioning services, and enhanced emergency-call functionality, as well as enhancements for downlink dual-layer beam-forming, see further below.

Recently, the main focus of 3GPP has turned towards the development of LTE release 10, further extending the performance and capabilities of LTE beyond release 8/9. An important aim of LTE release 10 is also to ensure that the LTE fulfills all the requirements for IMT-Advanced as defined by ITU $[2,3]$. The relation to IMT-Advanced is also the reason for the label "LTE-Advanced" sometimes given to LTE release 10 and beyond.

Spectrum flexibility is one of the key properties of the LTE radio-access technology. A wide range of different bandwidths is defined and both frequency division duplex (FDD) and time division duplex (TDD) modes-of-operation are supported allowing for operation in both paired and unpaired spectrum. An important requirement in the LTE design has been to avoid un-necessary fragmentation and strive for commonality between the FDD and TDD modes-of-operation. Not only does this increase the momentum in the definition and standardization of the technology but it also further improves the economy-of-scale of the LTE radio-access technology. This is important e.g. to ensure the availability of a wide-range of high-performance terminals and other devices supporting both FDD and TDD mode-of-operation.

At the same time, it is important that such an aim for commonality does not impose a constraint in terms of optimizing the performance of each duplex mode. It is e.g. important to ensure that duplex-specific properties can still be fully utilized. As an example, for TDD operation the available spectrum can be partitioned in the time domain between uplink and downlink in a flexible way and for this reason a range of different downlink/uplink configurations are supported [1]. It is further well known that TDD, due to the use of the same carrier frequency for uplink and downlink, may experience a higher degree of downlink/uplink channel reciprocity. The LTE radioaccess-technology has been designed to allow for extensive utilization of such reciprocity.

In this paper we describe the key characteristics of the LTE radio-access technology and its latest extensions in release 10, with special focus on the TDD mode-of-operation also known as TD-LTE. After a brief overview of the LTE radio-access technology in general, we discuss some specific characteristics and issues related to TDD operation. We also discuss some potential future evolution paths for the LTE radio-access technology, with specific focus on TD-LTE. Finally, we present some results from system-level evaluations showing that TD-LTE satisfies all requirements of IMT-Advanced as defined by ITU.

\section{OVERVIEW OF LTE RADIO-ACCESS}

\section{A. Basic transmission technology}

LTE is an OFDM-based radio-access technology, with conventional OFDM on the downlink and DFT-spread OFDM (DFTS-OFDM) [1] on the uplink. DFTS-OFDM allows for more efficient power-amplifier operation, thus providing the opportunity for reduced terminal power-consumption. At the same time, conventional OFDM avoids the need for receiverside equalization. The use of OFDM on the downlink combined with DFTS-OFDM on the uplink thus minimizes terminal complexity on the receiver side (downlink) as well as on the transmitter side (uplink), leading to an overall reduction in terminal complexity and power consumption. 


\section{B. Flexible bandwidth}

LTE supports a flexible transmission bandwidth up to $20 \mathrm{MHz}$ already in the first release (release 8). In release 10, the transmission bandwidth can be further extended by means of so-called carrier aggregation [4] where multiple component carriers are aggregated and jointly used for transmission to/from a single mobile terminal. Up to five component carriers can be aggregated allowing for transmission bandwidths up to $100 \mathrm{MHz}$.

In the general case, different number of component carriers can be aggregated for the downlink and uplink transmission directions respectively although, in case of TDD operation, symmetric aggregation can be expected to be the typical case at least from a network point-of-view. On the UE side, different carrier-aggregation capabilities for downlink and uplink are relevant also for TDD.

Spectrum aggregation is a special case of carrier aggregation where the aggregated carriers are located in different frequency bands. The possibility for spectrum aggregation is especially of value for operators with a fragmented spectrum situation, i.e. operators with access to a set of limited-sized spectrum pieces spread over different frequency bands. With spectrum aggregation, such operators can still provide high-data-rate services based on the availability of a wide overall bandwidth, even though they do not posses a single wideband spectrum allocation.

\section{Multi-antenna transmission}

A key feature of the LTE radio-access technology is the support for a rich set of multi-antenna transmission techniques. This includes downlink transmit diversity based on SpaceFrequency Block Coding (SFBC) for the case of two transmit antennas and SFBC in combination with Frequency Shift Time Diversity (FSTD) for four transmit antennas. In addition, downlink codebook-based pre-coding, including the possibility for multi-layer transmission (spatial multiplexing) with up to four layers, is supported in LTE release 8. This includes the possibility for rank-adaptation down to single-layer transmission, leading to codebook-based beam-forming, as well as a basic form of multi-user MIMO (SDMA)

The above mentioned multi-antenna techniques rely on cell-specific reference signals for demodulation. In addition, downlink single-layer pre-coding/beam-forming relying on UE-specific reference signals is also supported in release 8. UE-specific reference signals are pre-coded together with the data, implying that the pre-coder weights are not restricted to a certain codebook and do not need to be known to the receiver. In release 9, this non-codebook-based pre-coding and the corresponding UE-specific reference signals have been extended to support dual-layer transmission. One important application for transmission relying on UE-specific reference signals is beam-forming with more than four antennas.

In release 10 , the dual-layer beam-forming and the corresponding UE-specific reference signals are further extended to support transmission of to eight layers. Release 10 also defines so called CSI reference signals that can be used for enhanced channel-state-information feedback such as channel quality, rank indication and preferred pre-coding matrix.
On the uplink, only single-layer transmission is supported in release $8 / 9$ from a terminal perspective. However, uplink multi-user MIMO is supported in the sense that multiple users can be co-scheduled with the same bandwidth with orthogonal demodulation reference signals.

LTE release 10 further extends uplink multi-antenna transmission providing the possibility for uplink multi-layer transmission with up to four layers.

\section{Relaying functionality}

LTE-release 10 also extends the LTE radio-access technology with support for relaying functionality (Figure 1). With relaying, the mobile terminal communicates with the network via a relay node that is wirelessly connected to the remaining part of the radio-access network. The donercell/relay link is based on the LTE radio-interface technology and may operate on the same frequency as the relay/terminal link ("inband relaying") or on a different frequency ("outband relaying"). With the 3GPP "Type-1" relaying solution [4], the relay node will, from a terminal point-of-view, appear as an ordinary cell. This has the important advantage of simplifying the terminal implementation and making the relay node backwards compatible, i.e. accessible also to LTE release 8 terminals.
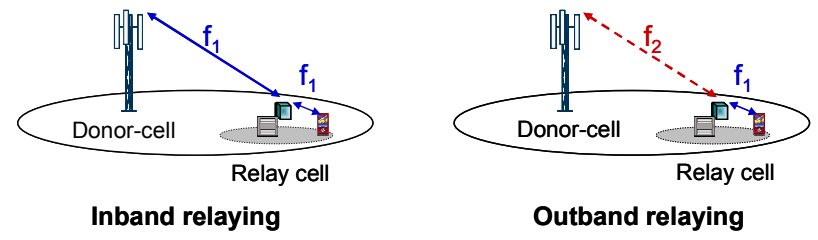

Figure 1. LTE relaying functionality

\section{TD-LTE SPECIFIC CHARACTERISTICS}

\section{A. Avoidance of duplex filter}

A benefit of TDD operation is the avoidance of a duplex filter in the mobile terminal. The duplex filter implies a nonnegligible complexity, especially for terminals supporting a large number of frequency bands. The duplex filter may also imply certain power losses in both the transmitter and receiver directions, power losses that are thus avoided in case of TDD operation.

On the other hand, TDD operation has a power disadvantage in the sense that maximum terminal power is typically specified as power in transmitted subframes, leading to a lower average transmit power due to the inherent reduced transmitter duty cycle of TDD. It should be noted that this power, and corresponding coverage, disadvantage is only relevant in power-limited scenarios.

\section{B. Channel reciprocity}

As already mentioned in the introduction, one key characteristic of TDD operation, with downlink and uplink transmission taking place in the same frequency band, is a higher degree of downlink/uplink channel reciprocity, 
compared to FDD operation where uplink and downlink transmission takes place on different carrier frequencies typically located relatively far from each other. It should be noted though that, also for TDD, only the radio channel is inherently reciprocal. To utilize the reciprocity, it must be ensured that the receiver and transmitter chains are also reciprocal e.g. by means of explicit $T X / R X$ calibration.

We note that uplink power control relies partially on channel reciprocity; there is an open loop component in the sense that a terminal will determine its uplink transmit power partially based on received signal power in the downlink. The extended channel reciprocity of TDD can be used beyond this as a tool to enhance different transmission technologies that are based on transmitter-side knowledge of more instantaneous and detailed channel properties. One prime example of this is downlink multi-antenna transmission.

As mentioned above, LTE includes support for UE-specific reference signals for demodulation of single- and dual-layer transmissions in release 8 and 9 respectively, and of up to eight layers in release 10. Since the UE-specific reference signals are pre-coded in the same way as the data, demodulation can be carried out without the terminal knowing exactly what precoder matrix has been applied at the transmitter side. This in turn means that the base station can freely choose the precoding or beam-forming weights without informing the terminal.

It is interesting to note that UE-specific reference signals for demodulation, which were first introduced in LTE release 8 to support beam-forming for TDD, has, for release 10 , been extended and become a major track for the evolution of LTE in general, i.e. for both FDD and TDD.

One advantage of exploiting channel reciprocity is that the standardizing effort is expected to be smaller as compared to approaches using explicit terminal feedback. Beam-forming with eight antennas is possible already in release 8 using channel reciprocity, whereas this will be possible with a codebook-based feedback approach first in release 10. Another advantage is that signaling overhead can be reduced. In fact, downlink beam-forming can be carried out with no feedback signaling overhead by using channel estimates from uplink transmissions that are anyway transmitted. Thus, assuming a high degree of channel reciprocity, non-code-book based precoding can, at least in principle, be carried out without explicit feedback of the channel-state from the receiver side. This impose though that there is a certain symmetry in the sense that there are transmissions in both uplink and downlink within the coherence time, and possibly within the coherence bandwidth.

Beyond release 10, multi-cell coordination (CoMP) techniques [4] can be considered, and exploitation of channel reciprocity is then a strong candidate to acquire channel state information to multiple cells.

\section{Interference between uplink and downlink}

Due to the use of the same frequency band for uplink and downlink transmission, TDD operation may lead to some additional interference scenarios that need to be taken care of in the radio-interface design and when deploying the system.
- Uplink-to-downlink interference where the uplink transmission of one terminal causes interference to a simultaneous downlink reception of another terminal

- Downlink-to-uplink interference where the downlink transmission at one base station causes interference to a simultaneous uplink reception at another base station.

These interference situations may occur within one operator (intra-operator interference) and then either within one carrier frequency or between carrier frequencies, where the former case is obviously more critical due to the absence of any frequency-domain filtering to suppress the interference.

The interference may alternatively occur between operators operating in frequency bands close to each other (interoperator interference).

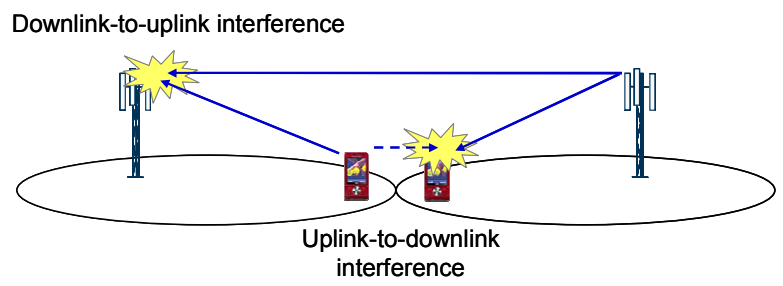

Figure 2. TDD-specific interference scenarios

The conventional way to handle/avoid the intra-operator interference is to apply time synchronization and use the same downlink/uplink configuration between cells as well as between carriers. In this way, uplink-to-downlink and downlink-to-uplink interference can be avoided, assuming a sufficiently large guard time between uplink and downlink transmissions. It should be noted that a relatively large guard time may needed as, especially in the case of downlink-touplink (base-station-to-base-station) interference, the interfering base stations may experience line-of-sight propagation conditions implying a possibility for substantial interference between base station also relatively far from each other. TD-LTE is designed with this in mind, allowing for a highly flexible guard time that can be adjusted to match different traffic situations and deployment scenarios.

Applying tight time synchronization and the same downlink/uplink configuration between operators, in order to avoid inter-operator interference, is obviously not as straightforward as within one operator. If tight time synchronization and coordinated downlink/uplink configurations are not possible, sufficiently large guard bands are needed between the operators. The required guard band depends on out-of-band-emission properties and the amount of interference that can be tolerated without significantly degrading system performance. This, in turn, depends on cell size, system load, base-station transmit power, etc. If timing synchronization and the same downlink/uplink configuration can be ensured between operators, the interference situation and corresponding guard-band requirements would be the same as for e.g. two FDD systems. 
Another very important issue regards co-existence between TD-LTE and the 3G TD-SCDMA radio-access technology. The TD-LTE time-domain structure has been chosen to match the TD-SCDMA time-domain structure thus allowing for coexistence between TD-LTE and TD-SCDMA without any specific guard-band requirements.

\section{FUTURE EVOLUTION OF TD-LTE}

LTE in general, and thus also TD-LTE, will continue to evolve in order to further enhance system performance and extend the capabilities of the radio-access technology. In this section we discuss some possible paths for this evolution. It should be noted that the details of the further evolution of LTE is still very much an open issue with no firm decisions yet taken.

\section{A. Exended flexibility in downlink/uplink assignment}

TD-LTE allows for a flexible downlink/uplink configuration in the sense that what set of subframes are used for downlink and uplink respectively is not fixed by the specification. However, the flexibility is limited in the sense that the downlink/uplink configuration is set at system start-up and is, in practice, not changed there-after. The reason and justification for this limited flexibility is an assumption that the downlink/uplink configuration must anyway be the same between all cells within a certain distance from each other in order to avoid severe downlink-to-uplink and/or uplink-todownlink interference, see above.

However, in certain situations there could be of value to be able to change the downlink/uplink configuration more dynamically, thus being able to more dynamically match the amount of available downlink and uplink resources to the instantaneous traffic situation.

One possible way to achieve this would be to define three types of subframes, downlink subframes, uplink subframes, and "flexible" subframes, compared to only downlink and uplink subframes in current TD-LTE releases. A terminal would receive on the downlink in the flexible subframes unless it has been explicitly or implicitly scheduled to transmit on the uplink in such a subframe.

Clearly, with a dynamic downlink/uplink configuration, there may be downlink-to-uplink and uplink-to-downlink interference between cells using instantaneously different configurations. One can envision two ways to handle this.

- Use fast information exchange between the cell sites to dynamically coordinate the scheduling, there-by avoiding downlink-to-uplink and uplink-to-downlink interference. This approach is mainly applicable to pico-cell deployments connected by high-speed backhaul to e.g. an overlaid macro site.

- Only utilize dynamic downlink/uplink assignment in low-load situations where inter-cell interference is less critical. It should be noted that future dense networks are expected to be data-rate limited rather than capacity limited. In such deployment scenarios, being able to dynamically assign as much resources as possible in a given transmission direction would be highly desirable. At the same time, the impact of the downlink-to-uplink and uplink-to-downlink interference on the system performance (overall capacity) would be less of an issue.

\section{B. Direct device-to-device communication}

One possible more long-term evolution paths would be to extend the LTE radio-access technology with support for direct device-to-device communication, i.e. make it possible for two mobile terminals to communicate directly with each other without going via the network.

One can envision two scenarios where the possibility for direct device-to-device communication could be of interest

- When no network infra-structure is available. This could e.g. be relevant for so-called National Safety and Protection Services (NSPS) scenarios.

- When network infra-structure is available but when communication directly between the terminals could be more efficient, either in terms of requiring fewer amounts of radio-resources for the same communication quality or allowing for improved communication quality, e.g. higher data rates. In this case, one could envision different degrees of network interaction in the device-to-device communication.

In case of direct device-to-device-communication, a terminal must obviously be able to receive on a frequency on which it normally transmits, alternatively be able to transmit on a frequency on which it normally receives. This hints at a benefit of TDD for direct device-to-device communication as, with TDD, the uplink and downlink frequencies coincide with a reduced impact on the mobile-terminal complexity as a consequence.

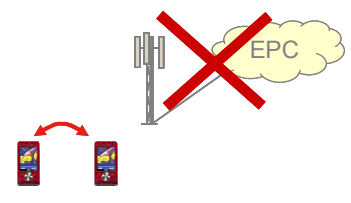

No network available

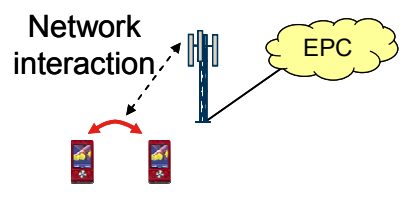

Network available
Figure 3. Direct device-to-device communication

\section{TD-LTE PERFORMANCE VS. IMT-ADVANCED REQUIREMENTS}

ITU has defined some basic requirements that have to be fulfilled by any IMT-Advanced technology [3]. Some of the most basic requirements, together with the corresponding capabilities of TD-LTE (from [5]), are summarized in Table 1.

Clearly, TD-LTE fulfills all the IMT-Advanced requirements. Actually, except for the bandwidth requirement (at least $40 \mathrm{MHz}$ ) and the requirement on uplink peak-spectral efficiency $(6.75 \mathrm{bps} / \mathrm{Hz})$, all IMT-Advanced requirements are fulfilled already with the first release of TD-LTE (release 8). What is needed beyond release 8 is the possibility for wider 
transmission bandwidth ( $>20 \mathrm{MHz}$ ) and the support for uplink multi-layer transmission (higher uplink peak spectral efficiency). Both these capabilities are included in TD-LTE release 10 .

TABLE I.

TD-LTE CAPABILITIES VS. IMT-ADVANCED REQUIREMENTS

\begin{tabular}{|l|c|c|}
\hline & $\begin{array}{c}\text { IMT-Advanced } \\
\text { requirement }\end{array}$ & TD-LTE \\
\hline Transmission bandwidth & At least $40 \mathrm{MHz}$ & Up to $100 \mathrm{MHz}$ \\
\hline $\begin{array}{l}\text { Peak spectral efficiency } \\
\text { - Downlink }\end{array}$ & $15 \mathrm{bps} / \mathrm{Hz}$ & $16.0[30.0]^{*} \mathrm{bps} / \mathrm{Hz}$ \\
- Uplink & $6.75 \mathrm{bps} / \mathrm{Hz}$ & $8.1[16.1]^{* *} \mathrm{bps} / \mathrm{Hz}$ \\
\hline $\begin{array}{l}\text { Latency } \\
\text { - Control plane }\end{array}$ & Less than $100 \mathrm{~ms}$ & $50 \mathrm{~ms}$ \\
- User plane & Less than $10 \mathrm{~ms}$ & $4.9 \mathrm{~ms}$ \\
\hline
\end{tabular}

* Value is for a $4 \times 4$ antenna configuration. Value in parentheses for $8 \times 8$

$* *$ Values is for a $2 \times 2$ antenna configuration. Value in parentheses for $4 \times 4$

Regarding more detailed requirements on average and celledge spectral-efficiency, 3GPP has carried out an extensive evaluation campaign to conclude on the performance of the LTE radio-access technology in relation to the IMT-Advanced requirements. An example of the TD-LTE system performance, for different environments (Indoor Hotspot, Urban Micro, Urban Macro, and Rural), are provided in the graphs of Figure 4 and Figure 5 below (from [6]). Once again, it can be noted that TD-LTE fulfills all the IMT-Advanced requirements. Detailed assumptions for the evaluations outlined in Figure 4 and Figure 5 can be found in [6].
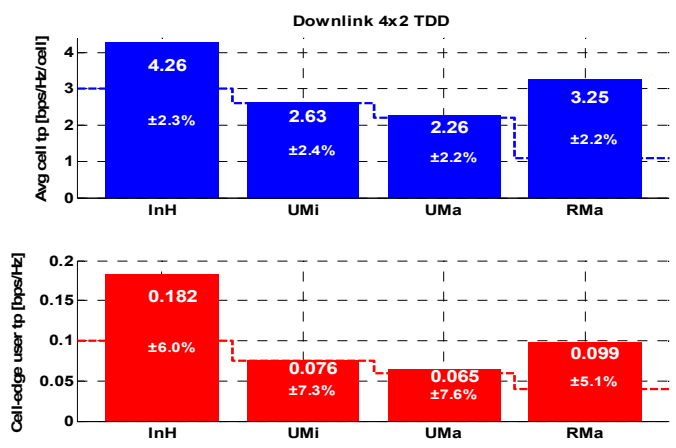

Figure 4. TD-LTE downlink average and cell-edge spectral efficiency. Dashed lines indicate the IMT-Advanced requirement.
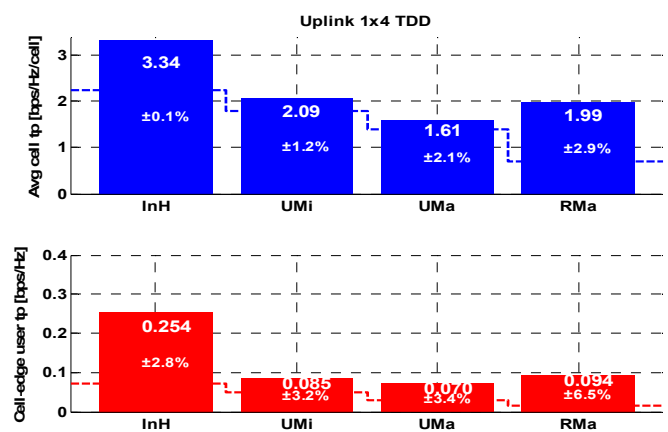

Figure 5. TD-LTE uplink average and cell-edge spectral efficiency. Dashed lines indicate the IMT-Advanced requirements.

These performance results are achieved without using any of the extended features of release 10. The IMT-Advanced requirements on average and cell-edge spectral efficiency can thus be fulfilled already with TD-LTE release 8 . It is important to point out that this does not mean that release 10 features, such as extended downlink multi-antenna transmission and relaying functionality, are of no use. Rather, these features take the capabilities of the TD-LTE radio-access technology even further, beyond IMT-Advanced. Thus, including more advanced features, such as extended multi-antenna transmission, the TDLTE system performance is further enhanced, beyond what is illustrated above.

\section{SUMMARY AND CONCLUSIONS}

This paper has discussed some specific benefits and issues related to the use of TDD duplex arrangement, specifically the TD-LTE radio-access technology. Some possible evolution paths for TD-LTE have been discussed. Finally, results from system-level performance evaluations of TD-LTE have also been presented, illustrating that TD-LTE fully satisfies the requirements on IMT-Advanced as defined by ITU.

Similarly to LTE/FDD being expected to be the dominating radio-access technology for mobile broadband for paired spectrum, TD-LTE is generally expected to be the dominating mobile-broadband radio-interface for unpaired spectrum.

\section{REFERENCES}

[1] E. Dahlman, S. Parkvall, J. Sköld, P. Beming, "3G Evolution: HSPA and LTE for Mobile Broadband", Academic Press, Oxford, UK, second edition, 2008

[2] ITU-R SG5, "Invitation for submission of proposals for candidate radio interface technologies for the terrestrial components of the radio interface(s) for IMT-Advanced and invitation to participate in their subsequent evaluation", Circular Letter 5/LCCE/2, March 2008

[3] ITU, "Requirements related to technical performance for IMT-Advanced radio interface(s)", ITU-R M.2134, http://www.itu.int/dms pub/itur/opb/rep/R-REP-M.2134-2008-PDF-E.pdf

[4] 3GPP TR 36.814, "Further advancements for E-UTRA (physical layer aspects"

[5] 3GPP TR 36.912, "Feasibility Study for Further Advancements of E-UTRA (LTE-Advanced)"

[6] A. Furuskär, "Performance Evaluations of LTE-Advanced - The 3GPP ITU proposal", The $12^{\text {th }}$ International Symposium on Wireless Personal Multimedia Communications (WPMC2009), Sendai, Japan, Sep. 2009. 